\title{
Solution of the Navier-Stokes Equations for a Two Dimensional Flow Field with Blocked Regions and Its Coupling with the Deposition Model "Michigan Wax Predictor"
}

\author{
Pedro-Alejandro-F. Becerra-Sierra, Diego-Fernando Bautista-Parada, \\ David-Alfredo Fuentes-Díaz, Arlex Chaves-Guerrero \\ Universidad Industrial de Santander \\ Cra 27 calle 9, Bucaramanga, Colombia \\ Pedro.Becerra1@correo.uis.edu.co; diego.Bautista@correo.uis.edu.co dfuentes@uis.edu.co; achavesg@uis.edu.co
}

\begin{abstract}
Due to the low temperatures of subsea water and the presence of waxes in the crude-oil, wax deposition might occur inside pipelines; causing transport problems; leading to economic problems for the petroleum industry and possible environmental disasters in the sea. One of the leading premises in most of the current wax deposition models is to only consider the axial component of the velocity in conservation equations. The wax deposition is a process proved to be driven by radial mass transport; therefore, these assumptions are presumed to reduce accuracy. The inclusion of the radial velocity component will affect the deposit thickness and mass fraction predicted results. The model assumes molecular diffusion as the primary mechanism of transport. The flow field is obtained by solving the momentum equations for the radial and axial velocities using the finite volume method and the SIMPLE method on a colocated structured grid. All the simulations were done under laminar flow conditions.
\end{abstract}

Keywords: CFD, Wax Deposition, Petroleum, Heat Transfer, Mass Transfer, Modeling.

\section{Introduction}

The solid deposition process is a common problem in many industries such as fouling in heat exchangers [1], coke deposition in coking reactors [10], and wax deposition in crude-oil transport pipelines[9, 7, 11]. This deposition causes problems just as the reduction of the effective heat transfer, and the plugging of pipelines. Both difficulties imply an increase in operational costs and the introduction of new risks for the process due to the maintenance of the equipment.

The offshore crude-oil extraction platforms have become more popular every day, hence, making the study of crudeoil transport through submarine pipelines a matter of great importance, especially, the transport problems attributed to the wax deposition phenomena. Crude-oil is a mixture of many components, including asphaltenes and paraffin or waxes, which are mainly responsible for the difficulties that arise in the extraction and transport processes [11]. The wax deposition on the internal surface of these pipes is a common phenomena in transport processes where the temperatures of the surroundings are near $4^{\circ}$ Celsius (however, depending of the crude-oil and circumstances, deposition might be present in onshore transport as well).

When the crude-oil flows from the well through the pipelines it suffers a heat loss due to the low temperature of the sub-sea water, resulting in a decrease of its temperature, probably to values inferior to its wax appearance temperature (WAT) [2], reducing the wax's solubility, and finally, causing their precipitation and deposition on the surface [4]. In spite of the fact that preventive and corrective methods have been proposed, the risk of performing them without the necessary information about the wax deposit (localization, thickness, hardness) can lead to an incident [5].

\section{Model Development}

The Michigan Wax Predictor (MWP) is a deposition model developed by the University of Michigan Porous Media Research Group, originated from the work of Singh et. al., in 2000 [15]. This model accounts for the aging of the deposit and predicts successfully the thickness of it. This model has been validated by many experimental studies [16, 7, 8, 12], and has been extended to turbulent flow. Due to its selection of deposition mechanism and accurate assumptions, it is considered a good base for this work. 


\subsection{Flow Field Prediction}

The wax deposition is a process proved to be driven by radial mass transport [9]. Therefore, the assumption made by the MWP model of a parabolic velocity profile could be correct in order to reduce both the complexity of the momentum equations needed to solve and the time necessary to do it. However, this assumption is presumed to reduce the accuracy of the predicted results thickness and mass fraction of the deposit. To deal with this, this work aims to extend the MWP equations to a two dimensional flow field by solving the following equations:

$$
\begin{gathered}
\rho\left(V_{r} \frac{\partial V_{r}}{\partial r}+V_{z} \frac{\partial V_{r}}{\partial z}\right)=-\frac{\partial P}{\partial r}-\left[\frac{1}{r} \frac{\partial}{\partial r}\left(r \tau_{r r}\right)+\frac{\partial \tau_{z r}}{\partial z}-\frac{\tau_{\theta \theta}}{r}\right] \\
\rho\left(V_{r} \frac{\partial V_{z}}{\partial r}+V_{z} \frac{\partial V_{z}}{\partial z}\right)=-\frac{\partial P}{\partial z}-\left[\frac{1}{r} \frac{\partial}{\partial r}\left(r \tau_{r z}\right)+\frac{\partial \tau_{z z}}{\partial z}\right]
\end{gathered}
$$

The contribution of this work to the base model is the inclusion of the two-dimensional flow field, for this task, the development of a computational algorithm able to solve the Navier-Stokes equations in cylindrical coordinates is essential. This work presents the results of the algorithm for laminar cases along with the other approaches used.

The method proposed uses a collocated mesh to store the transport-related variables and the SIMPLE method to solve the velocity-pressure coupling. The equations are discretized using the finite volume method (FVM). The explicit time scheme is used, and a comparison between the hybrid scheme and the roe's SUPERBEE [14] scheme is presented.

\section{Results}

The algorithm used to solve the Navier-Stokes equations is tested on the benchmark problem known as lid driven cavity (LDC). The hybrid scheme is employed for three cases changing the Reynolds number between 100, 400 and 1000. A structured computational grid of $100 \times 100$ for a total of 10000 cells is employed; the results are presented and compared with the results obtained by Ghia [6] in figure 1.

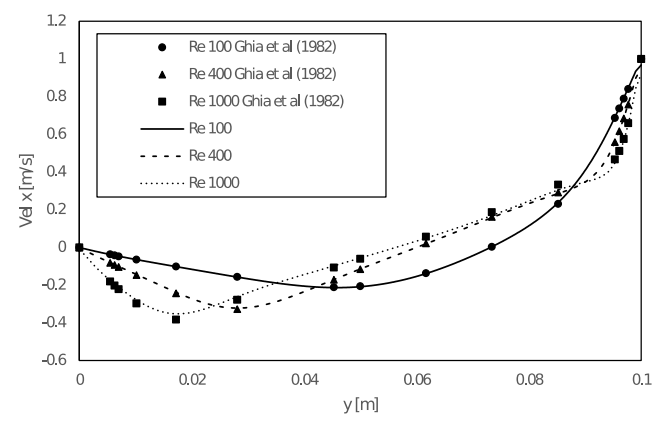

a)

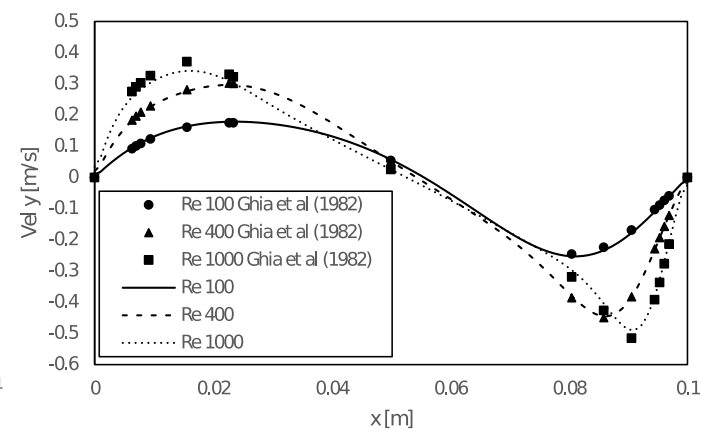

b)

Fig. 1: Velocities for the LDC using the hybrid scheme. (a) x-component along a vertical line through the geometric center. (b) ycomponent along a horizontal line through the geometric center.

The average error for the velocity in the $\mathrm{x}$-component is $6 \%$, and $9.8 \%$ for the $\mathrm{y}$-component. The simulation time required to accomplish a residue of $1 \times 10^{-9}$ is 13 minutes. Although the results are good, the behavior of a high order scheme such as the roe's SUPERBEE is analyzed for the Reynolds number of 1000, (the reason for this comparison is the hybrid scheme increased error for higher Reynolds numbers). For this case a structured mesh of $72 \times 72$ for a total of 5184 elements was employed; the results are shown in figure 2. 


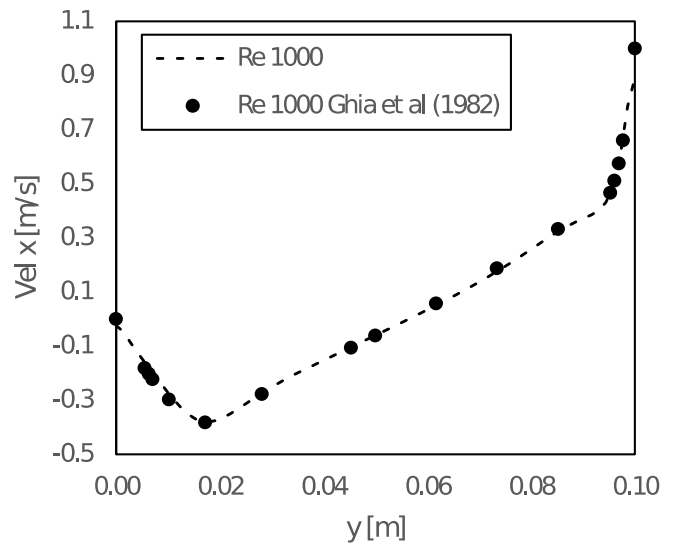

a)

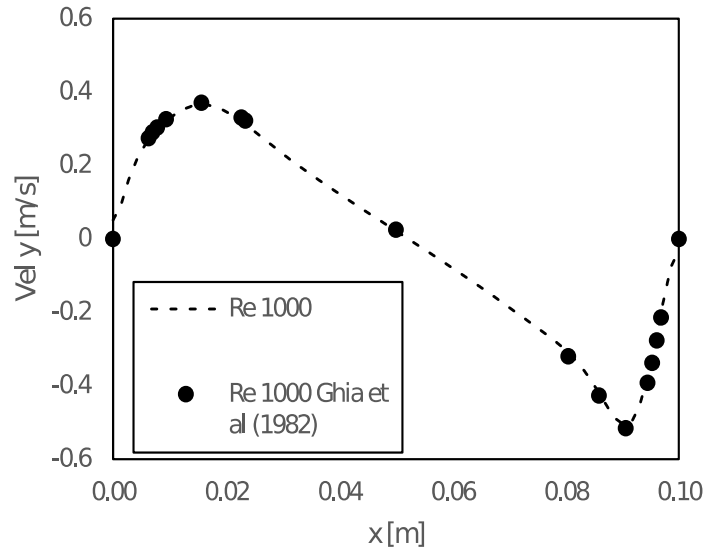

b)

Fig. 2: Velocities for the LDC using the roe's SUPERBEE scheme. (a) x-component along a vertical line through the geometric center. (b) y-component along a horizontal line through the geometric center.

The average error for the velocity in the $\mathrm{x}$ coordinate is $5.11 \%$, and $3.56 \%$ for the $\mathrm{y}$-component. The simulation time required to accomplish a residue of $1 \times 10^{-9}$ is 5 minutes. The advantage of using a high order scheme can be noted by the fact that a lower error can be accomplished employing a computational grid of almost half of the elements of the hybrid scheme case in $38.45 \%$ of the time.

The second part of the presented method consists of the prediction of the flow field in the presence of the deposit. Once the deposit is created, a radial component of the velocity will appear, and the flow field must be recalculated with the assumption of a zero velocity in the solid part of the computational domain. Due to the complexity of generating a new mesh to fit the fluid domain each time the deposit grows and the flow field is altered, the Blocked-off Regions approach proposed by Patankar [13] is tested as an alternative, using only one computational grid. The flow inside a duct with a converging section [3] was analyzed. The axial-symmetry presented allowed for the simulation of only half the duct. The computational domain consist of a rectangle, however, the original geometry is presented next in figure 3.

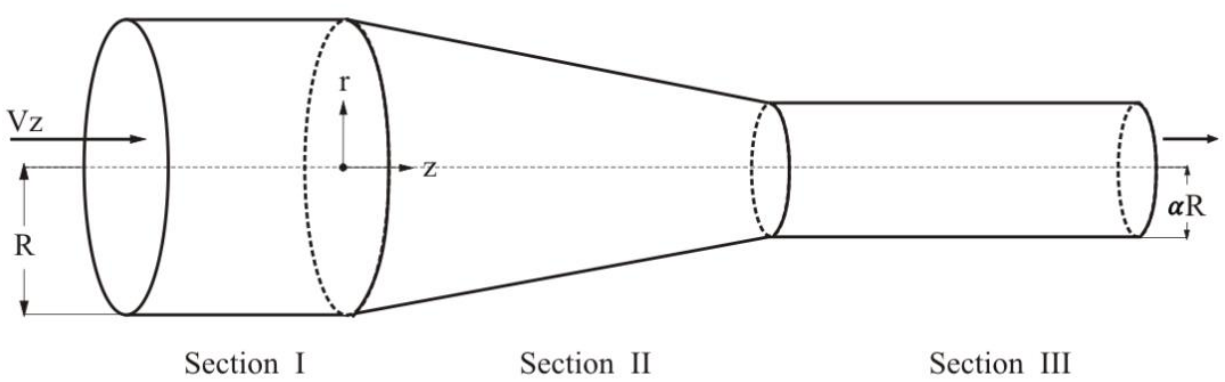

Fig. 3: Duct with a converging section (Adapted from Bird et. al., (1987) []ㅡ).

The results for each section of the duct are presented and compared with the analytical solution proposed by Bird et. al., [3] in figure 4. 


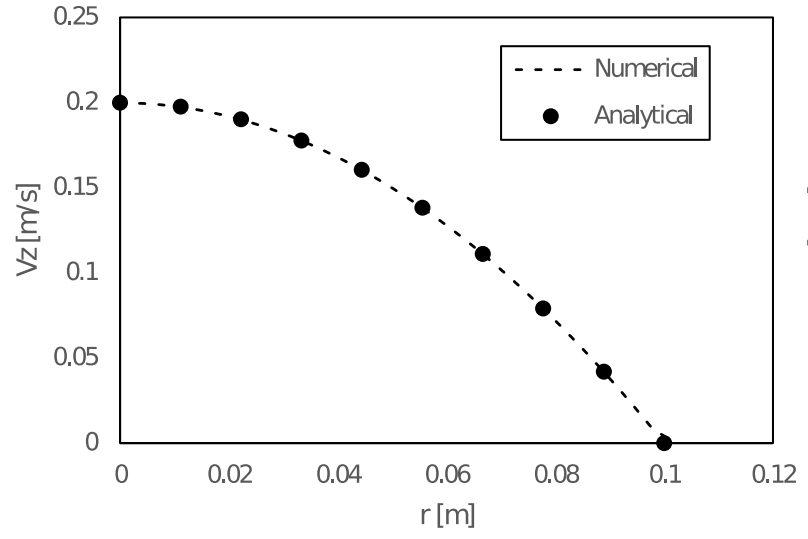

a)

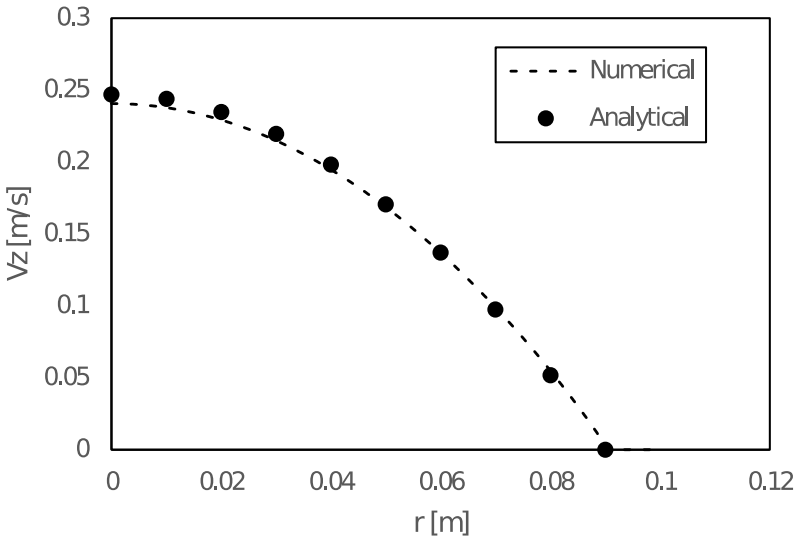

b)

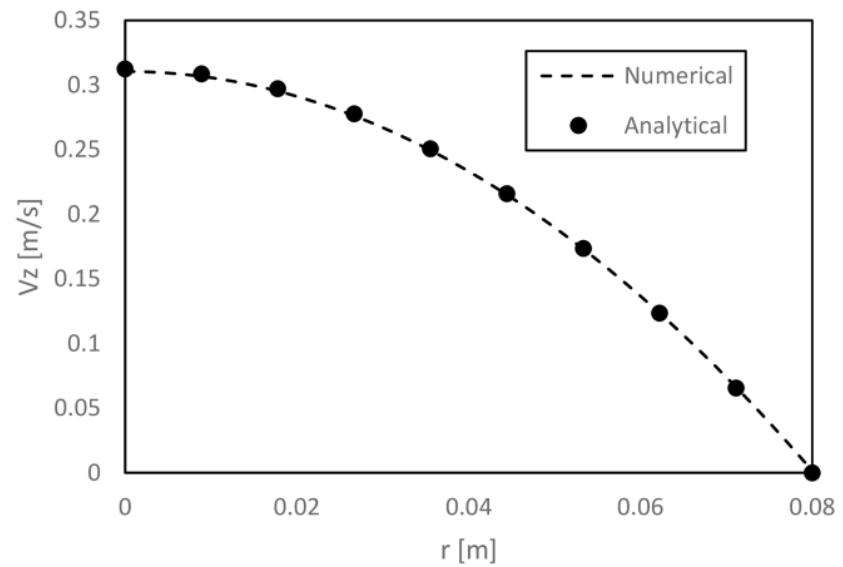

c)

Fig. 4: Comparison of the axial velocity between the Blocked-off regions approach and the analytical solution. a) Section I b) Section II c) Section III.

It can be noted in section II that the velocity near the center of the pipeline differs from the analytical solution, this error can be attributed to the interpolation of values along the boundary from interior points in the post-processing stage.

\section{Conclusions}

The solution of the Navier-Stokes equations in cylindrical coordinates for a two dimensional flow field has been successful. The results obtained are accurate and the error obtained can be minimized using a high order scheme such as the roe's SUPERBEE. The setting of the value for the velocity inside the elements corresponding to the deposit (solid region) using the Blocked-Off Regions approach has been successfully implemented and proved as seen in figure 4, thus, the need of creating a new mesh each time the domain changes, has been surpassed, and the flow field has been obtained correctly. Now that the algorithm for the flow field is complete and validated, this work moves forward to integrate these equations into the heat and mass transfer equations with the objective of predicting the thickness and mass fraction of the deposit, and analyzing the radial velocity effect on them.

\section{References}

[1] M. Bayat, J. Aminian, M. Bazmi, S. Shahhosseini, K. Sharifi, "CFD modeling of fouling in crude oil pre-heaters," Energy Conversion and Management, vol. 64, pp. 344-350, 2012.

[2] A. Berne-Allen Jr, L. T. Work, "Solubility of Refined Paraffin Waxes in Petroleum Fractions," Industrial \& Engineering Chemistry, vol. 30, pp. 806-812, 1938.

[3] R. Byron, Robert C. Bird, O. H. Armstrong, Dynamics of polymeric liquids, Fluid mechanics. 1987. 
[4] C. E. Reistle Jr., O. C. Blade, Paraffin and Congealing Oil Problem. 1932.

[5] G. Fung, W. P. Backhaus, S. McDaniel, M. Erdogmus, "To Pig or Not to Pig: The Marlin Experience with Stuck Pig," Offshore Technology Conference, 2006.

[6] U. Ghia, K. N. Ghia, C. T. Shin, "High-Re solutions for incompressible flow using the Navier-Stokes equations and a multigrid method," Journal of Computational Physics, vol. 48, no. 3, pp. 387-411, 1982.

[7] Z. Huang, H. S. Lee, M. Senra, H. S. Fogler, "A fundamental model of wax deposition in subsea oil pipelines," AIChE Journal, vol. 57, no. 11, pp. 2955-2964, 2011.

[8] Z. Huang, M. Senra, R. Kapoor, H. S. Fogler, "Wax deposition modeling of oil/water stratified channel flow," AIChE Journal, vol. 57, no. 4, pp. 841-851, 2011.

[9] Z. Huang, S. Zheng, H. S. Fogler, Wax Deposition: Experimental Characterizations, Theoretical Modeling, and Field Practices. 2015.

[10] P. J. Ellis, E. E Hardin, "How petroleum delayed coke forms in a drum," Light Met (Warrendale, Pa.), vol. 1993, pp. 509-515, 1992.

[11] H. S. Lee, "Computational and Rheological Study of Wax Deposition and Gelation in Subsea Pipelines," Ph.D. Dissertation, Chemical Eng., University of Michigan, Michigan, 2008.

[12] Y. Lu, Z. Huang, R. Ho, L. Amundsen, H. S. Fogler, "Counterintuitive Effects of the Oil Flow Rate on Wax Deposition," 2012.

[13] S. Patankar, Numerical Heat Transfer and Fluid Flow. 1980.

[14] P. Roe, "Characteristic-Based Schemes for the Euler Equations," Annual Review of Fluid Mechanics, vol. 18, no. 1, pp. 337-365, 1986.

[15] P. Singh, Gel deposition on cold surfaces. 2000.

[16] R. Venkatesan, H. S. Fogler, "Comments on analogies for correlated heat and mass transfer in turbulent flow," AIChE Journal, vol. 50, no. 7, pp. 1623-1626, 2004. 